\title{
Nexus between green bonds, financial, and environmental indicators
}

\author{
Ngo Thai Hung* \\ University of Finance-Marketing,Ho Chi Minh City, Vietnam
}

Received: 4 November 2020

Revised: 2 April 2021

Accepted: 2 April 2021

\begin{abstract}
This study uses a recent approach to examine the causal associations between green bonds and other conventional assets, including clean energy, price of $\mathrm{CO} 2$ emission allowances, Bitcoin, and the S\&P 500 stock market covering from January 2013 to March 2019. We find a bidirectional relationship between green bonds, S\&P 500, and Bitcoin markets, while green bonds have a unidirectional connection with the price of $\mathrm{CO} 2$ emission allowances. More importantly, clean energy has a significant impact on green bonds and vice versa throughout the sample period. The results have several useful implications for green bond investors in connection with portfolio design and hedging decisions.
\end{abstract}

Keywords: green bonds; equity and other prices; portfolio diversification; causality JEL Classification Codes C58, G10, G12, Q50

\section{Introduction}

The emergence of green bonds connected with environmentally friendly projects and the top product of cryptocurrency offers more investment strategies (Le et al. 2020; Hung, 2020; Park et al. 2020). Transitioning toward a low-carbon economy requires a significant investment of long-term financial resources and the exploitation of suitable financial instruments for that purpose. Compared with traditional bonds, green bonds are a distinctive, sustainability-oriented, fixed-income financial instrument to increase funds earmarked by the issuer for environmentally friendly projects in line with a climate-resilient economy (Reboredo, 2020; Agliardi and Agliardi, 2019). More precisely, green bonds provide distinctive environmental benefits because of their green nature, like low-carbon, energy-efficient, and climate-friendly (Huynh et al. 2020a; Park et al. 2020; Sartzetakis, 2020). Therefore, raising funds through a large bond market that can give capital, could be suitable in the long run. For investors, green bonds are stable investments that offer long-term income.

Green bonds can be examined by considering the general properties of bonds, and longstanding research interest regarding the bond market is its association with the equity market. The volatility spillover mechanism is transferred between the bond, and other traditional assets play a prominent role in portfolio composition and risk management (Huynh, 2020;

\footnotetext{
* Corresponding author. E-mail: hung.nt@ufm.edu.vn.
}

Citation: Hung, N. T. (2021) Nexus between green bonds, financial, and environmental indicators, Economics and Business Letters, 10(3), 191-199.

DOI: 10.17811/ebl.10.3.2021.191-199 
Huynh et al. 2020a). Since green bonds are a kind of bond, studying the interrelatedness between the green bond and other related assets would help global investors organize their portfolios. The green bonds just began to be actively traded recently; academic research on this topic is insufficient (Park et al. 2020; Reboredo et al. 2020; Hachenberg and Schiereck, 2018). Therefore, the current study represents meaningful conclusions about the intercorrelation between green bonds and other asset classes.

Regarding the interplay between green bonds and other conventional financial markets, Liu et al. (2020) put forward a positive dynamic average and tail dependence between green bonds and clean energy markets. Reboredo et al. (2020) find a strong relationship between green bonds and treasury and corporate bonds in the short and long run. Specifically, green bonds are weakly connected with stock and energy assets at various time scales. Similarly, Hammoudeh et al. (2020) report a significant causality running from the US 10-year Treasury bond to green bonds, and $\mathrm{CO} 2$ emission allowances price has a considerable impact on green bonds during the research period. More importantly, the authors provide evidence that there is a low relationship between the clean energy index and green bonds. Baulkaran (2019) indicated that the nexus between green bonds and stock returns is positive and significant. This finding implies that shareholders see this form of financing as value-enhancing, and funds from green bonds issuance are a means of risk mitigation. In the same year, Broadstock and Cheng (2019) provide evidence that the relationship between green and black bonds is sensitive to changes in financial market volatility, economic policy uncertainty, daily economic activity, and oil prices.

In the context of the 4th industrial revolution, Huynh et al. (2020b) and Le et al. (2020) indicate that Bitcoin and gold significantly influence green bonds and NASDAQ AI. More importantly, Le et al. (2020) reveal that Bitcoin is a net contributor to volatility shocks while green bonds are net receivers. Similarly, Park et al. (2020) center on the volatility dynamics and transmissions between equity and green bond markets and document that the causal relationship between the green bonds and equity markets is statistically significant. However, the two markets have several volatility spillover effects.

In the light of conscious investors' interests in environmental risk, they pay close attention to green bonds as a social responsibility commitment (Nguyen et al. 2020). Many nations pledged to transition to a climate-resilient economy-green bond market, which could thrive (Reboredo et al. 2020). Additionally, green bonds offer a robust opportunity to diversify their investments by using an alternative to conventional assets (Nguyen et al. 2020). Therefore, understanding price spillover between green bonds and other financial assets is significant to identify green bonds' performance and their helpfulness for hedging and managing portfolio risks (Hammoudeh et al. 2020; Reboredo et al. 2020; Jakubik and Uguz, 2020; Hung, 2021).

In general, most investors believe a homogeneous influence of green bonds on other conventional financial markets. This study report distinguishes other asset classes by divergent time responses to innovations in the market. We examine the influence of lag-period green bonds on other asset classes using a novel methodology, namely Multilayer Perceptron Neural Network Non-linear Granger causality and Transfer Entropy. These specifications outperform other traditional economic models used previously by considering asymmetric and non-linear impacts (Huynh et al. 2020b; Huynh, 2020). This is the paper employing these new methodologies to explore the research purpose to the best of our knowledge. Therefore, such estimations would localize the causal relationships running between green bonds and the examined variables. Moreover, they give straightforward insight into investors operating in the green bond markets, especially in price forecasts, portfolio diversification, and risk management.

Due to these advantages, in this paper, we attempt to assess the degree of market intercorrelation and shock transmission between green bonds and other related financial markets, particularly considering S\&P 500, Bitcoin, clean energy, and emission prices since these markets 
are an appropriate choice for investment. Even though green bonds use the same pricing determinants as any other conventional financial assets, they start to finance environmentally beneficial projects, which generates how to price oscillations in financial markets that may impact the non-pecuniary motives. Therefore, we focus on the causal association between the green bond market and some conventional financial assets by considering how contemporaneous and lagged price changes are transmitted, which might play a vital role in an investment portfolio.

There are some contributions that this study provides to the existing literature. First, centering on the intercorrelation between green bonds and other financial markets, the present study reveals evidence of applying green bonds as a potential diversifier to stock or commodity portfolios. It attracts environment preference investors and general investors. Apart from the economic perspective, this study also contributes recent methods such as Multilayer Perceptron Neural Network Non-linear Granger causality and Transfer Entropy for explaining the causal association between green bonds and other assets. Our findings supplement previous studies mainly on cross-asset nexus and affirm the hedging potential of green bonds for other asset markets.

We find a bidirectional relationship between green bonds, S\&P 500, and Bitcoin markets, while green bonds have a unidirectional connection with the price of $\mathrm{CO} 2$ emission allowances. More importantly, clean energy has a significant impact on green bonds and vice versa throughout the sample period.

The rest of the paper is as follows. Section 2 depicts data and briefly introduces the methodologies used. Section 3 discusses the empirical results. Section 4 represents the conclusion.

\section{Data}

This paper investigates the interplay between the green bond and financial asset classes from January 2013 to March 2019. It comprises five series, namely the S\&P Green Bond index (GB), to demonstrate the market of green bonds. Similarly, our dataset consists of daily prices of the Clean Energy Index (CEI), CO2 emission allowances (EP), Bitcoin price (BIT), and S\&P 500 (SP). Specifically, the CO2 emission allowance price corresponds to the spot price of the European Union $\mathrm{CO} 2$ emission allowances collected from European Energy. We construct our analysis using the S\&P GB index to demonstrate the global GB market (Reboredo, 2018). This index contains only those bonds whose proceeds are used to fund environmentally friendly projects. The sample period is determined by the data's availability on the S\&P GB (Hammoudeh et al. 2020). The data are collected from Datastream, and the estimation is conducted using returns computed by taking logarithm difference.

Table 1. Descriptive statistics of the analyzed variables.

\begin{tabular}{lrrrrr}
\hline \hline \multicolumn{1}{c}{ GB } & EP & CEI & SP & BIT \\
\hline Mean & -0.008680 & 0.076596 & 0.027142 & 0.042418 & 0.331146 \\
Maximum & 1.478259 & 40.44985 & 4.558178 & 4.840324 & 30.85640 \\
Minimum & -2.392061 & -43.47355 & -4.965323 & -4.184256 & -34.53162 \\
Std. Dev & 0.331630 & 5.217703 & 1.062679 & 0.812541 & 4.629399 \\
Skewness & -0.353796 & -0.010188 & -0.197509 & -0.482628 & -0.602605 \\
Kurtosis & 5.963517 & 14.10089 & 4.858943 & 6.615646 & 13.81530 \\
Jarque-Bera & $624.2898^{* * *}$ & $8287.224^{* * *}$ & $242.8874^{* * * *}$ & $941.8104^{* * * *}$ & $7963.965^{* * * *}$ \\
ADF & $-42.09778^{* * *}$ & $-37.12764^{* * *}$ & $-32.23318^{* * *}$ & $-40.83942^{* * *}$ & $-38.97992^{* * *}$ \\
\hline \hline
\end{tabular}

Notes: ${ }^{* * * *}$ denotes rejection of null hypotheses at the $1 \%$ level of significance.

Figure 1. Correlation among returns series under investigation. 


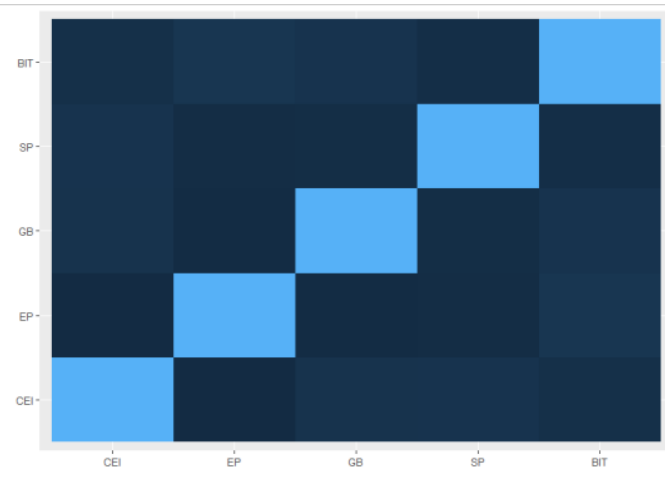

(a) Pearson correlation

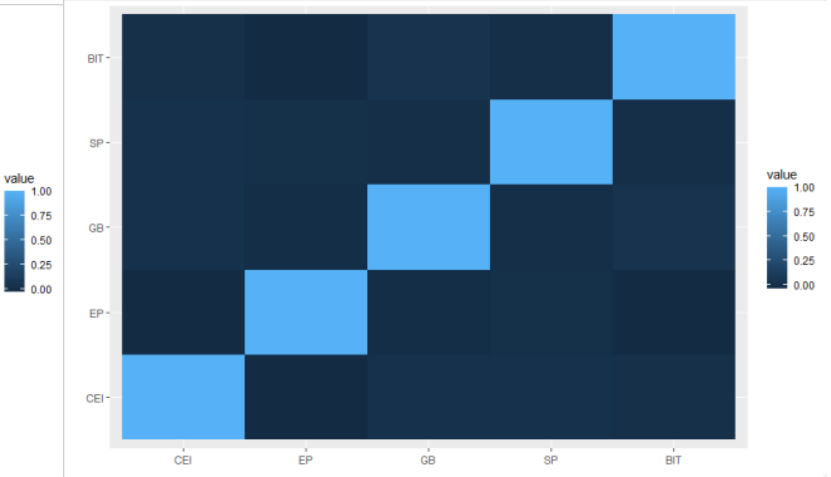

(b) Spearman correlation

Figure 2. Plots of the distribution and correlation structure of GB and other asset's returns.

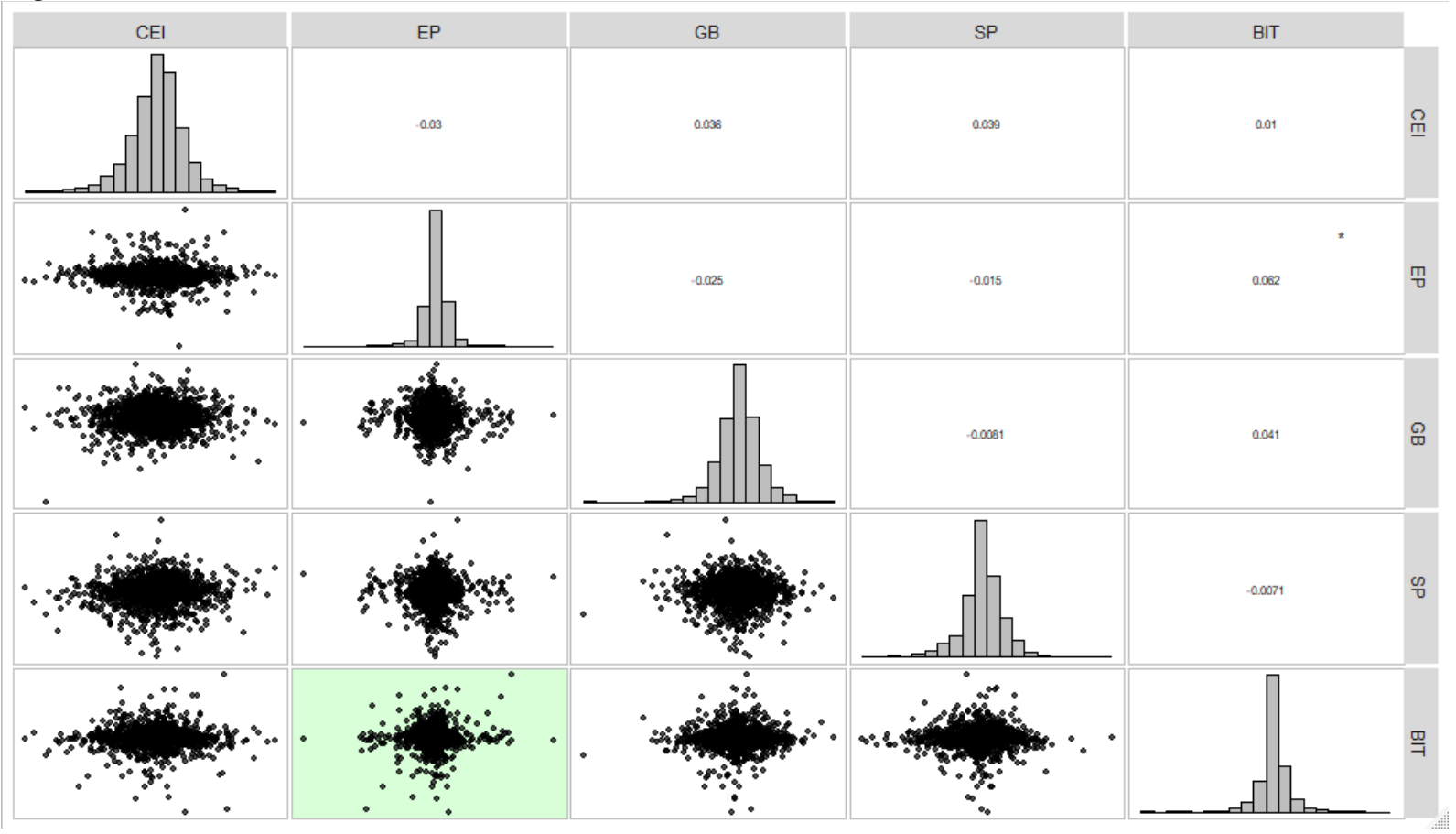

Table 1 documents the descriptive statistics for log returns. All returns series do not have a normal distribution in connection with the Jarque-Bera test for normality. The augmented Dickey-Fuller test shows that our data are stationary and appropriate for further analysis.

Figure 1 displays the correlation of our variables based on the Pearson and Spearman approaches. As shown in Figure 1, the connection between the green and other asset classes is significantly weak. Besides, there is a considerable interrelatedness between GB and Bitcoin and S\&P 500 markets. This means that the GB market has a similar directional co-movement. According to Huynh et al. (2020b), one of the drawbacks of applying Pearson correlation is based on linear symmetric and parametric comparison. Nevertheless, all the returns series are not normally distributed, as indicated in Table 1. The Spearman correlation illustrates that our variables under study have a stronger connection than the Pearson correlation shows. Figure 2 exhibits the overall distribution of the data and the pairwise correlations between the asset returns, which provides us further insight into the data distribution and correlation structure. 


\section{Methodology}

We examine the relationship between GB index, Clean Energy Index (CEI), CO2 emission allowances (EP), Bitcoin price (BIT), and S\&P 500 (SP) by using both advanced techniques: Multilayer Perceptron Neural Network Non-linear Granger causality and Transfer Entropy. Unlike several conventional approaches, these models allow us to make more precise estimates, following Huynh's papers (2020). More specifically, non-linear Granger causality allows us to overcome some difficulties such as linear assumption, autoregressive vector conveyance, and normal distribution (Huynh, 2020; Hung, 2019).

There are some advantages to using Multilayer Perceptron Neural Network Non-linear Granger causality to detect our research's connectedness. First, the issues of non-linear interdependencies of variables can be addressed. Second, a neural network can help us to find out causal associations without non-linear additive effects. To complement the Multilayer Perceptron Neural Network Non-linear Granger causality test, we employ Transfer Entropy specification to capture the information flows about the direction of a variable with respect to time, which would show co-movement between the GBs and other asset returns. More precisely, the causal relationship would be clear because the main advantage of using Transfer Entropy is the information transmission dimension (Huynh et al. 2020b).

\section{Multilayer perceptron neural network non-linear Granger causality}

Let $x_{t} \in \mathbb{R}^{p}$ denote a p-dimensional stationary time series. Granger causality is typically studied using the vector autoregression model (VAR), which $x_{t}$ is a linear combination of the past K lags of the series.

$$
x_{t}=\sum_{k=1}^{K} A^{(k)} x_{t-k}+\varepsilon_{t}
$$

where $A^{(k)}$ is the $(p \times p)$ matrix that specifies how $\mathrm{K}$ lags impacts the future evolution of the series and $\varepsilon_{t}$ is mean zero noise.

A non-linear autoregressive model allows $x_{t}$ to evolve according to more general non-linear dynamics.

$$
x_{t}=g\left(x_{(t-1)}, \ldots, x_{(t-K)}\right)+e_{t}=\left(g_{1}\left(x_{(t-1)}, \ldots, x_{(t-K)}\right), \ldots, g_{p}\left(x_{(t-1)}, \ldots, x_{(t-K)}\right)\right)^{T}+
$$

where $g_{i}$ is a continuous function that specifies how the past $\mathrm{K}$ lags impacts series $i$.

This study uses the simple schematic algorithm for MLP called component-wise. We model $g_{i}$ with a separate MLP so that impacts from inputs to outputs are easier to disentangle. Assume, for each $i, g_{i}$ takes the form of an MLP with L layers, and the vector $h_{t}^{l}$ denotes the values of the $l$ th hidden layer at a time $t$. Let $W=\left\{W^{1}, \ldots, W^{L}\right\}$ denote the weights at each layer and let the first layer weights be written as $W^{1}=\left\{W^{11}, \ldots, W^{1 K}\right\}$. The first hidden values at a time $t$ are given by:

$$
h_{t}^{l}=\sigma\left(\sum_{k=1}^{K} W^{1 k} x_{t-k}+b^{l}\right)
$$

where $\sigma$ is an activation function and $b^{l}$ is the bias at layer $l$. The output, $x_{t i}$, is given by

$$
x_{t i}=w_{O}^{T} h_{t}^{L}+\varepsilon_{t}
$$

where $w_{O}^{T}$ is the output decoder and $h_{t}^{L}$ is the final hidden output from the final $\mathrm{L}^{\text {th }}$ layer.

MLP creates our output variables, which are employed to the VAR case to capture the causal relationship.

$$
\min _{W}\left(\sum_{k=1}^{K}\left(x_{t i}-g_{i}\left(x_{(t-1):(t-K)}\right)\right)^{2}+\lambda \sum_{j=1}^{p}\left\|\left(W_{: j}^{11}, \ldots, W_{: j}^{1 K}\right)\right\|_{F}\right)
$$

For large enough $\lambda$, the solutions to Eq. (5) will lead to many zero columns in each $W^{1 k}$ matrix, suggesting only a small number of estimated Granger causal connections.

\section{Transfer entropy}


Let $i_{t}^{(k)}=\left(i_{t}, \cdots, i_{t-k+1}\right) h_{J}(l)$ as analogously for process J. Kullback and Leibler (1951) define the generalized Markov process as follows:

$$
p\left(i_{t+1} \mid i_{t}^{(k)}\right)=p\left(i_{t+1} \mid i_{t}^{(k)}, j_{t}^{(k)}\right)
$$

The escort distribution $\phi_{q}(j)=\frac{p^{q}(j)}{\sum_{j} p^{q}(j)}$ with $q>0$ to normalize. Transfer entropy can be written as:

$$
T E_{X \rightarrow Y(k, l)}=\frac{1}{1-q} \log \left(\frac{\sum_{j} \phi_{q}\left(i_{t}^{(k)}\right) p^{q}\left(i_{t+1} \mid i_{t}^{(k)}\right)}{\sum_{i, j} \phi_{q}\left(i_{t}^{(k)}, j_{t}^{(k)}\right) p^{q}\left(i_{t+1} \mid i_{t}^{(k)}, j_{t}^{(k)}\right)}\right)
$$

The Renyi entropy with the Markov bootstrap and repeated the bootstrap with the escort distribution was employed by Dimpfl and Peter (2013), which is used in this paper.

\section{Results}

Table 2 reports the bidirectional association between the GB and other asset returns with five lag days. We select a five-period lag because the GB has the same properties as equities assets, with five trading days. As a result, the lag illustrates the influence of one determinant on another in the trading week. Also, the definition of Multilayer Perceptron Neural Network Non-linear Granger causality may impact the present period of the indicator variable when doing historical data. Hence, this approach does not estimate the current influence (lag 0) of the indicator variable on the dependent variable. Transfer Entropy might tackle the contemporaneous and previous influence. This is the main reason why the current study employs both to explore the divergent perspective.

It is clear from Table 2 that there is a bidirectional connectedness between EP, SP, BIT, and GBs. This means that innovations in the GB may give rise to innovations in the equity markets and vice versa. On the other hand, the green bond has unidirectional impacts on $\mathrm{CO} 2$ emission prices. More precisely, the green bond is sensitive to the volatility of the other asset classes. These results are in full agreement with Hammoudeh et al. (2020). Our paper also extends the existing literature (Reboredo et al. 2020; Le et al. 2020) regarding the interdependence between the green bonds and other conventional asset classes using a new approach, Multilayer Perceptron Neural Network Non-linear Granger causality. Further, the results also confirm that a price spillover effect between these assets and the green bond exists, which helps investors. This reveals that investors should include some of these assets in their equity portfolios for diversification.

Table 2. The interrelatedness between the green bonds and other financial assets.

\begin{tabular}{crrrrr}
\hline \hline Causal direction & Lag 1 & Lag 2 & Lag 3 & Lag 4 & Lag 5 \\
\hline$G B \rightarrow C E I$ & 0.5794 & 0.6957 & -0.6195 & -0.3224 & -0.3843 \\
$C E I \rightarrow G B$ & -0.3550 & -2.2676 & -0.3269 & $2.3524^{* * *}$ & -1.289 \\
$G B \rightarrow E P$ & $1.2724^{*}$ & 0.0744 & $1.6787^{* * * *}$ & -2.77991 & $4.1398^{* * * *}$ \\
$E P \rightarrow G B$ & -1.1612 & -14.9464 & $2.1719^{* * *}$ & -5.8547 & -3.4350 \\
$G B \rightarrow S P$ & $3.4650^{* * *}$ & $7.8309^{* * *}$ & $7.3538^{* * *}$ & -2.3104 & -0.1795 \\
$S P \rightarrow G B$ & -0.2268 & 0.7135 & $13.182^{* * *}$ & 0.0587 & -1.5261 \\
$G B \rightarrow B I T$ & -3.04893 & -1.1078 & $1.4461^{* *}$ & -1.5328 & -0.05283 \\
$B I T \rightarrow G B$ & -0.0035 & $0.4189^{*}$ & $6.1722^{* * *}$ & 0.7507 & 0.44932 \\
\hline \hline
\end{tabular}

Notes: $:^{* * *, * * * *}$ reveal the significance at $10 \%, 5 \%$ and $1 \%$, respectively. The null hypothesis is that no causal association in the specific lag period. The optimal lag length for the VAR model is selected using the Akaike's Information Criterion (AIC).

Table 3. The impact of Green bond on traditional financial assets. 


\begin{tabular}{lr}
\hline \hline Casual direction & GB \\
\hline$\rightarrow C E I$ & $0.0035[0.0020]$ \\
$C E I \rightarrow$ & $0.0070^{*}[0.0016]$ \\
$\rightarrow E P$ & $0.0040[0.0021]$ \\
$E P \rightarrow$ & $0.0056[0.0019]$ \\
$\rightarrow S P$ & $0.0043[0.0020]$ \\
$S P \rightarrow$ & $0.0053[0.0018]$ \\
$\rightarrow B I T$ & $0.0062[0.0022]$ \\
$B I T \rightarrow$ & $0.0065^{* *}[0.0021]$ \\
\hline \hline
\end{tabular}

Notes: ${ }^{* * *}$ reveal the significance at $10 \%$ and $5 \%$, respectively. Standard errors of the related coefficients are represented in square brackets. The results of main values are defined as the Shannon transfer entropy with $\mathrm{n}=300$ while the number of shuffles are selected 100 observations at random.

We estimate transfer entropy values, and the results are reported in Table 3. Differing from Multilayer Perceptron Neural Network Non-linear Granger causality, transfer entropy does not present the directional and signal interplay as correlations or coefficients. Interestingly, we can observe that the GB's contemporary influence on other asset classes is relatively minimal. Specifically, the GBs are likely to have a relationship with the Bitcoin market and the clean energy index. This result also complements the finding by Le et al. (2020). Apart from that, the results suggest that $\mathrm{CO} 2$ emission prices and S\&P 500 stock returns seem to be independent by not receiving any impact from the GB market. Overall, transfer entropy estimation shows a significant information flow from the Bitcoin and S\&P 500 markets to GBs. These results are also consistent with Hammoudeh et al. (2020), Huynh et al. (2020b), and Park et al. (2020).

In sum, we found that there is a causal association between the GB and other asset classes under consideration. Our analysis is robust with two techniques, which took into account the price spillover effects. More importantly, we find that the co-movement of the clean energy index and CO-2 emission prices are somewhat independent of the green bond using the transfer entropy framework. Likewise, we find strong relationships between green bonds, Bitcoin, and clean energy. The interplay attained a significant value and remained somewhat stable throughout the two estimations. From an economic perspective, these results are in line with Reboredo et al. 2020 and Nguyen et al. (2020), who demonstrated that commodities' financialization caused a considerable relationship between green bonds, commodities, and stock markets. Also, there exists a strong correlation between clean energy and green bonds over the period shown. Green bonds would be considered a vital diversification asset when adding to the investment portfolios with other asset classes. Besides, the findings indicate that the green bonds market significantly impacts the price of $\mathrm{CO} 2$ emission allowances. These returns increase with carbon pricing, which generates a level playing field for low-carbon investments over high-carbon alternatives. As a result, a sufficiently high $\mathrm{CO} 2$ price supports the successful market introduction of green bonds (Hammoudeh et al. 2020). Nevertheless, we can expect green bonds to thrive if carbon pricing takes off. The causal association between the value of green bonds and carbon prices may be crucial for emissions trading systems.

\section{Conclusion}

Accelerating the transition to a low-carbon economy requires raising funds for green projects, which give new green finance opportunities for investors. Green bonds are becoming increasingly complicated in a sustainable development-oriented financial market. Since investors start to evaluate price environment risks, a systematic understanding of the price spillover between the green bond and other markets plays an important role in shaping the 
risk-return properties of green bonds and the transition to sustainable economic and social development.

Contributing to the existing literature, we investigate the causal associations between green bonds and other assets, including clean energy, price of $\mathrm{CO} 2$ emission allowances, cryptocurrency, and the S\&P 500 stock market, seeking hedging characteristics of green bonds. The novelty of this paper lies in centering on the interrelatedness between green bonds and other assets. The interrelatedness provides evidence of using green bonds and a potential diversifier in the stock or commodities portfolio. It also sheds light on the causal association between the GB and other assets mentioned above using Multilayer Perceptron Neural Network Non-linear Granger causality and Transfer Entropy approaches. This paper also fills the literature gap by contributing new specifications for forecasting the causal nexus between green bonds and other assets under examination, which extends investors' knowledge of different assets above.

Our empirical result reveals a bidirectional relationship between green bonds, stock, and Bitcoin markets, while green bonds have a unidirectional connection with the price of $\mathrm{CO} 2$ emission allowances. More importantly, clean energy has a significant impact on green bonds and vice versa throughout the sample period. The results have several useful implications. First, evidence of substantial connectedness of green bonds with stock, Bitcoin, clean energy, and price of $\mathrm{CO} 2$ emission allowances provide shelter to the price oscillations in these markets. Our result gives straightforward insight into green bonds. This new instrument enhances the transition to a low-carbon economy and makes more diversified strategies for investors for risk hedging. Put differently, the allocation across green bonds, stock, Bitcoin, and commodities might provide benefits to investors. The estimations also provoke policymakers and financial managers' attention to reducing risks and innovation transmission between various financial markets. Therefore, it is crucial to enhance policies supporting the development of the green bonds market.

\section{References}

Agliardi, E., and Agliardi, R. (2019) Financing environmentally-sustainable projects with green bonds, Environment and development economics, 24(6), 608-623.

Broadstock, D. C., and Cheng, L. T. (2019) Time-varying relation between black and green bond price benchmarks: Macroeconomic determinants for the first decade,. Finance Research Letters, 29, 17-22.

Baulkaran, V. (2019) Stock market reaction to green bond issuance, Journal of Asset Management, 20(5), 331-340.

Dimpfl, T. and Peter, F. J. (2013) Using transfer entropy to measure information flows between financial markets, Studies in Nonlinear Dynamics \& Econometrics, 17 (1), 85102.

Huynh, T. L. D., Hille, E., and Nasir, M. A. (2020b) Diversification in the age of the 4th industrial revolution: The role of artificial intelligence, green bonds and cryptocurrencies, Technological Forecasting and Social Change, 159, 120188.

Huynh, T. L. D., Nasir, M. A., Vo, V. X., and Nguyen, T. T. (2020a) "Small things matter most": The Spillover effects in the cryptocurrency market and Gold as a silver bullet, The North American Journal of Economics and Finance, 101277.

Huynh, T. L. D. (2020) The effect of uncertainty on the precious metals market: New insights from Transfer Entropy and Neural Network VAR, Resources Policy, 66, 101623.

Hung, N. T. (2021) Volatility Behaviour of the Foreign Exchange Rate and Transmission Among Central and Eastern European Countries: Evidence from the EGARCH Model, Global Business Review, 22(1), 36-56. 
Hung, N. T. (2020) Time-Frequency Nexus Between Bitcoin and Developed Stock Markets in the Asia-Pacific, The Singapore Economic Review, 1-26.

Hung, N. T. (2019) Equity market integration of China and Southeast Asian countries: further evidence from MGARCH-ADCC and wavelet coherence analysis, Quantitative Finance and Economics, 3(2), 201-220.

Hachenberg, B., and Schiereck, D. (2018) Are green bonds priced differently from conventional bonds?, Journal of Asset Management, 19(6), 371-383.

Hammoudeh, S., Ajmi, A. N., and Mokni, K. (2020) Relationship between green bonds and financial and environmental variables: A novel time-varying causality, Energy Economics, 92, 104941.

Jakubik, P., and Uguz, S. (2020) Impact of green bond policies on insurers: evidence from the European equity market, Journal of Economics and Finance, 1-13.

Kullback, S., and R. A. Leibler. (1951) On Information and Sufficiency, The Annals of Mathematical Statistics, 22 (1), 79-86.

Liu, N., Liu, C., Da, B., Zhang, T., and Guan, F. (2020) Dependence and risk spillovers between green bonds and clean energy markets, Journal of Cleaner Production, 279, 123595.

Le, T. L., Abakah, E. J. A., and Tiwari, A. K. (2020) Time and frequency domain connectedness and spill-over among fintech, green bonds and cryptocurrencies in the age of the fourth industrial revolution, Technological Forecasting and Social Change, 162, 120382.

Nguyen, T. T. H., Naeem, M. A., Balli, F., Balli, H. O., and Vo, X. V. (2020) Time-frequency comovement among green bonds, stocks, commodities, clean energy, and conventional bonds, Finance Research Letters, 101739.

Park, D., Park, J., and Ryu, D. (2020) Volatility Spillovers between Equity and Green Bond Markets, Sustainability, 12(9), 3722.

Reboredo, J. C., Ugolini, A., and Aiube, F. A. L. (2020) Network connectedness of green bonds and asset classes, Energy Economics, 86, 104629.

Sartzetakis, E. S. (2020) Green bonds as an instrument to finance low carbon transition, Economic Change and Restructuring, 1-25. 